Editorial

\title{
Acknowledgment to Reviewers of Fire in 2020
}

\section{Fire Editorial Office}

Citation: Fire Editorial Office. Acknowledgment to Reviewers of Fire in 2020. Fire 2021, 4, 3.

https://doi.org/10.3390/fire4010003

Published: 31 January 202

Publisher's Note: MDPI stays neutral with regard to jurisdictional claims in published maps and institutional affiliations.

Copyright: (C) 2021 by the author Licensee MDPI, Basel, Switzerland. This article is an open access article distributed under the terms and conditions of the Creative Commons Attribution (CC BY) license (http:// creativecommons.org/licenses/by/4.0 /).
MDPI AG, St. Alban-Anlage 66, 4052 Basel, Switzerland

Peer review is the driving force of journal development, and reviewers are gatekeepers who ensure that Fire maintains its standards for the high quality of its published papers. Thanks to the cooperation of our reviewers, in 2020 the median time to first decision was 13.4 days and the median time to publication was 2.5 days. The editors would like to express their sincere gratitude to the following reviewers for their precious time and dedication, regardless of whether the papers were finally published:

\begin{tabular}{|c|c|}
\hline Abbate, Andrea & Curran, Timothy \\
\hline Almeida, Miguel & Czymzik, Markus \\
\hline Ambrosia, Vincent G. & Davies, G. Matt \\
\hline Anderson, Kerry & Deshmukh, Dhananjay Kumar \\
\hline Anderson, Lysanna & Diaz-Hernandez, Jose Luis \\
\hline Ascoli, Davide & Diendorfer, Gerhard \\
\hline Bajocco, Sofia & Dimyadi, Johannes \\
\hline Baker, Kirk & Duan, Weili \\
\hline Baker, Sarah & Duine, Gert-Jan \\
\hline Baker, William & Duvall, Chris S. \\
\hline Barker, Jason & Edgeley, Catrin \\
\hline Barrio, Aitor & Eftekharian, Esmaeel \\
\hline Barry, Allison & Farguell, Angel \\
\hline Beckett, Heath & Fernandes, Paulo \\
\hline Benito, Alfonso Alonso & Fernandez, Helena \\
\hline Bento-Gonçalves, António & Filippi, Jean-Baptiste \\
\hline Bilbao, Bibiana & Fowler, Cynthia \\
\hline Blackhall, Melisa & Frank, Graham S. \\
\hline Blake, David & Furlaud, James \\
\hline Boisrame, Gabrielle & Gałaj, Jerzy \\
\hline Boucher, Jonathan & Gallagher, Michael \\
\hline Bowman, David & Ganteaume, Anne \\
\hline Boychuk, Den & Gao, Xiulin \\
\hline Brzezińska, Dorota & García-Delgado, Carlos \\
\hline Buchwald, Andrea & Gollner, Michael \\
\hline Buriticá, Susana Rodríguez & Gomes, Letícia \\
\hline Caballero, David & Goncharov, Anton \\
\hline Calvo, Leonor & Goodrick, Scott L. \\
\hline Campanharo, Wesley Augusto & Hagan, Donald \\
\hline Campbell, Michael & Hamman, Sarah T. \\
\hline Casula, Marcello & Hanan, Erin \\
\hline Charney, Jay & Hanson, Nicholas J. \\
\hline Cho, Jaeil & Hashempour, Javad \\
\hline Cisek, Marcin & Hassan, Quazi K. \\
\hline Clements, Craig & He, Yinan \\
\hline
\end{tabular}


Coates, Adam

Costafreda-Aumedes, Sergi

Hilton, James

Hohner, Amanda

Hossain, Akter

$\mathrm{Hu}$, Xiaoqin

Hudak, Andrew

Hunter, Molly E.

Iniguez, Jose M.

Innangi, Michele

Jamnik, Veronica

Jandl, Robert

Jia, Shenyue

Jiménez-Ruano, Adrián

Johnson, Ed

Johnston, James D.

Johnston, Lynn

Just, Michael

K. Velez, Anne-Lise

Kalabokidis, Kostas

Kane, Van R.

Karns, Jameson

Kartsios, Stergios

Kinoshita, Alicia M.

Kolden, Crystal

Krasovskiy, Andrey

Krix, Daniel W.

Kuligowski, Erica

Kuligowski, Erica D.

Lanorte, Antonio

Lao, Seng Kin

Laris, Paul

Lee, Eric

Levine, Carrie

Lindley, Todd

Log, Torgrim

Logan, Timothy

Lopes, António M.Gameiro

Luc Dupuy, Jean

M. Barton, Andrew

M. Condé, Tiago

Madrigal, Javier

Maingi, John

Manzello, Samuel

Marchese, Francesco

Marshall, Ginny

Martínez, Juan Ramón Molina

Martinka, Jozef

Mast, M. Alisa

Mataveli, Guilherme Augusto Verola

McBride, Steven
Hedayati, Faraz

Heinsch, FaithAnn

Melchiorre, Andrea

Metallinou, Maria-Monika

Milenković, Milan

Miller, Eric

Mindykowski, Pierrick

Mohammadzadeh, Ali

Molinas-González, Carlos R.

Monokrousos, Nikolaos

Monteiro, Alexandra

Moosmuller, Hans

Mora, Jose R.

Moran, Christopher J.

Mota, Bernardo

Mouillot, Florent

Mukhortova, Liudmila

Narapusetty, Balachandrudu

Nielsen-Pincus, Max

Nik-Bakht, Mazdak

Nolan, Rachel

Novara, Agata

Nunes, A. N.

Östman, Birgit

Pansing, Elizabeth

Parsons, Russ

Paugam, Ronan

Pausas, Juli

Penney, Greg

Picotte, Josh

Pimont, François

Pokorný, Jiří

Pokswinski, Scott

Prichard, Susan J.

Prior, Lynda

Pyne, Stephen

Quan, Xingwen

Rahman, Md. Habibur

Rakowska, Joanna

Raposo, Jorge Rafael Nogueira

Raptis, Dimitrios

Regos, Adrián

Reszka, Pedro

Rhodes, Edward

Righini, Gaia

Roberts, Gareth

Rodman, Kyle

Ruecker, Gernot

Ruffault, Julien

Sà, Ana

Sacks, Jason D. 
McGranahan, Devan Allen

McGrattan, Kevin B.

Sayaka, Suzuki

Scholes, Robert

Seielstad, Carl A.

Sharma, Sonisa

Silva Junior, Celso Henrique Leite

Silva, Francisco Rodríguez Y

Smith, Kevin T.

Sommers, William T.

Stasiewicz, Amanda

Steelman, Toddi A.

Strack, Maria

$\mathrm{Su}, \mathrm{Ye}$

Talhelm, Alan F.

Talucci, Anna

Tanner, Benjamin

Thomas-Van Gundy, Melissa

Thompson, Matt

Torero, Jose Luis

Torikai, Hiroyuki
Saito, Yoko

Sakellariou, Stavros

Trucchia, Andrea

Tsiourlis, Georgios

Vander Yacht, Andrew

Varner, J. Morgan

Varner, Morgan

Vigilante, Tom

Wagtendonk, Jan Van

Waigl, Christine

Wang, Qingsong

Węgrzyński, Wojciech

Wester, David B.

White, Angela M.

Wilkinson, Sophie

Williams, Roger

Wollstein, Katherine

Wrona, Paweł

Xofis, Panteleimon

Yoder, Jonathan

Yurkonis, Kathryn 